\title{
La Política Fiscal en la encrucijada. El caso de América Central $^{1}$
}

Autores: Gabriel Castellanos, Jorge Cornick, Héctor Pérez Brignoli, Pablo Rodas, Carlos Acevedo, Maynor Cabrera, Nikken Cullman, Manuel Delgado y Jonathan Menkos

\section{Resumen Ejecutivo}

\section{Antecedentes}

138 La política fiscal en América Central enfrenta serios problemas. Los niveles actuales de inversión en infraestructura y de gasto público en educación, salud y seguridad son claramente insuficientes frente a lo que se requiere para impulsar un proceso sostenido de crecimiento con bienestar y equidad en un contexto de globalización y democracia. Verificar de manera sistemática, pública y comprensiva la eficacia con que se (a) utilizan los recursos públicos en el

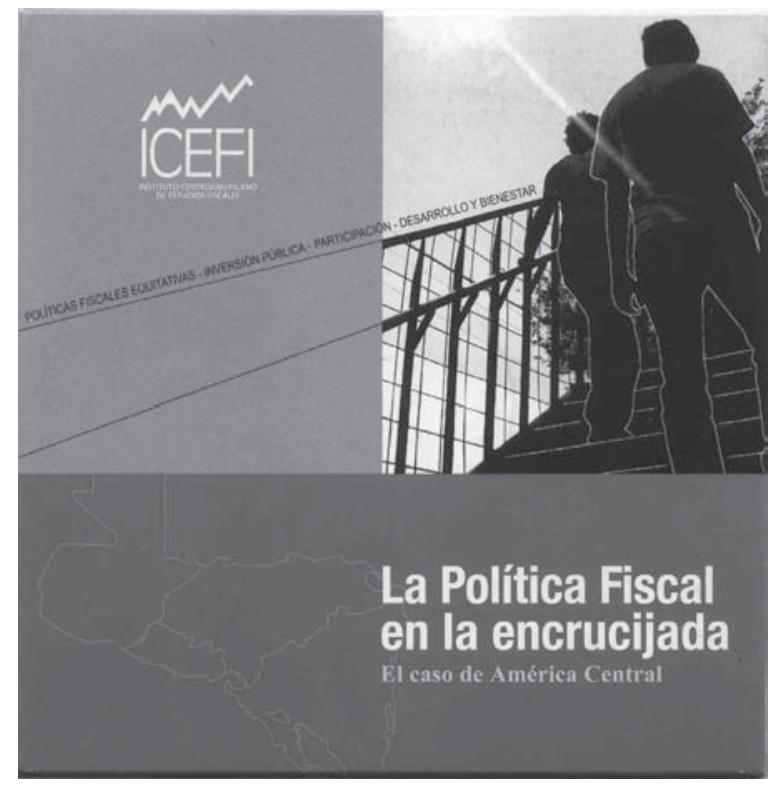
ámbito nacional y local para alcanzar los resultados y las metas de desarrollo es todavía una asignatura pendiente. A su vez, la transparencia del gasto, especialmente en el ámbito municipal o descentralizado, deja mucho que desear y son comunes las acusaciones de corrupción y de mal uso de los recursos públicos. Las actividades de los organismos de contraloría son deficientes en la mayor parte de países. Además, los poderes legislativos tienen dificultades para asegurar una fiscalización que vaya más allá de las denuncias de corrupción.

Los ingresos de los gobiernos de los países del istmo son insuficientes para cubrir el gasto que se necesita. El control de los evasores y las sanciones para castigarlos todavía son extremadamente débiles. No se aprovecha el potencial de los impuestos aplicados a los ingresos o al patrimonio. Los impuestos aplicados al consumo continúan siendo la

1 Texto basado en la presentación realizada por el Dr. Juan Alberto Fuentes, Director Ejecutivo del ICEFI, el 23 julio de 2007 en Managua. El evento fue co-organizado por el Instituto Nicaragüense de Investigaciones y Estudios Tributarios (INIET) que dirije el Dr. Julio Francisco Báez, miembro del Consejo Asesor del ICEFI. 
base de la tributación en la mayor parte de países del istmo, en contraste son otros países que cuentan con dos pilares, uno de impuestos aplicados al consumo y otro de impuestos aplicados a los ingresos. Parte de esta debilidad se explica por un conjunto de exenciones y exoneraciones que erosionan seriamente la base de todos los impuestos, pero principalmente del impuesto sobre la renta.

La historia demuestra que los obstáculos que impiden cambiar esta situación han sido formidables. La oposición fuerte y concentrada de ciertas élites económicas no permitió que se desarrollara un sistema fiscal más complejo y adecuado. En épocas más recientes algunas de estas élites han invertido en sectores privilegiados que no pagan impuestos y, por consiguiente, se oponen a que se fortalezca la tributación a pesar de que estos sectores son los más dinámicos de la economía. La existencia de estos privilegios, junto con el hecho de que amplios sectores de la población centroamericana de altos y bajos ingresos, no perciben que se benefician de los servicios del Estado, hace difícil alcanzar acuerdos o contratos sociales que le den un sustento político de largo plazo a las finanzas públicas.

El establecimiento de regímenes democráticos en todos los países ha abierto espacios para que reformas fiscales se lleven a cabo, aunque con dificultades. Persiste, en los partidos políticos, una dependencia financiera de los sectores económicos poderosos; y persiste una debilidad técnica en materia fiscal, especialmente tributaria. Además, la ausencia de mayorías parlamentarias dificulta la implementación rápida de reformas y el tema tributario parece no enfrentarse con la seriedad que amerita.

\section{Avances de la política fiscal}

A pesar de que persisten serios problemas, ha habido importantes avances que permiten vislumbrar cambios en el futuro. La gestión y transparencia del gasto público, especialmente del gasto de los gobiernos centrales, ha mejorado notablemente en los últimos años. La introducción de sistemas integrales de administración financiera, con información abierta al público en varios países, lo demuestra. El gasto público para impulsar el desarrollo social, especialmente en la educación, ha aumentado significativamente en todos los países. La mayor parte de este gasto está incidiendo de manera positiva en el bienestar de los hogares más pobres. Aunque los resultados de la implementación de diversas estrategias de reducción de la pobreza pueden ser polémicos, programas de atención primaria universal en salud, educación y otros complementarios, que han transferido recursos a familias pobres o vulnerables a condición de que envíen a sus hijos a la escuela y participen en programas de salud materno-infantil, han tenido incuestionables resultados positivos donde se han aplicado.

Los avances en el ámbito tributario también han sido importantes. Mayores tasas de crecimiento económico le han permitido a las administraciones tributarias contar con un mayor costo absoluto de recursos. La carga tributaria, que mide la proporción del ingreso nacional que se destina al pago de impuestos, también ha aumentado en todos los países. Este progreso refleja reformas parciales, generalmente poco dramáticas, pero que han permitido reducir algunos privilegios, fortalecer la administración tributaria y disminuir la evasión. La introducción de sistemas de control y retención ha contribuido a aumentar la 
recaudación en algunos países, especialmente del IVA, mientras que otras micro-reformas, incluyendo definiciones más amplias del ingreso gravable o más precisas de los gastos deducibles, han dado lugar a una mayor recaudación del impuesto sobre la renta.

Los poderes legislativos, mediante diversos tipos de alianzas, han logrado introducir estas reformas, aun con la oposición de ciertas élites económicas, y la búsqueda de "pactos fiscales" formales o informales ha comenzado a ganar terreno en todos los países del istmo. La preocupación con el tema fiscal es manifiesta. Las reformas recientes más orientadas a lograr la "equidad horizontal", es decir, que los que tienen ingresos o niveles de consumo similares paguen niveles de impuestos parecidos, son las que más han avanzado. Además, el fortalecimiento de la administración tributaria, con un fuerte apoyo de la comunidad internacional, ha puesto de manifiesto su potencial para realizar esfuerzos ambiciosos de movilización de recursos.

\section{La encrucijada: la posibilidad de hacerle frente a los desafíos}

Los avances en el ámbito del gasto público y de tributación establecen fundamentos sólidos para poder enfrentar y resolver los problemas que aún persisten. Son la base para plantear que América Central se encuentra en una encrucijada que presenta buenas posibilidades de avanzar mucho más. En la encrucijada actual pueden identificarse al menos seis desafíos en el ámbito del gasto público y de la gestión del presupuesto y cinco desafíos en el ámbito tributario, además de un gran desafió, político, de impulsar las reformas con efectividad. Todos ellos se enmarcan dentro del necesario desarrollo de la institucionalidad de cada país y del istmo en su conjunto, incluyendo tanto el fortalecimiento de normas y reglas como el esfuerzo de los organismos responsables de asegurar su implementación.

\section{a) Los desafíos de la gestión del presupuesto y del gasto público}

Los seis desafíos por el lado del gasto público y la gestión del presupuesto incluyen, primero, mejorar la transparencia aumentando el acceso público a la información disponible y reforzando las atribuciones, desempeño y credibilidad de las contralorías, especialmente en algunos países. Segundo, corresponde mejorar la gestión y planificación del presupuesto, introduciendo presupuestos multianuales ampliamente debatidos e indicadores de desempeño con vista a establecer, gradualmente, un presupuesto de resultado que privilegie la sencillez para facilitar una gestión hábil del presupuesto. Tercero, corresponde avanzar con la descentralización del gasto público, pero resolviendo previamente los problemas de falta de transparencia, de débil rendición de cuentas y de corrupción, que a menudo existen este ámbito. La movilización de recursos propios, especialmente mediante impuestos aplicados a la propiedad inmueble o predial, debería ser parte esencial de este esfuerzo.

Cuarto, conviene realizar un gran esfuerzo por aumentar la inversión en infraestructura, especialmente en aquellas áreas o sectores donde los plazos de retribución y las condiciones existentes no facilitan alianzas público-privadas que conduzcan a que el sector privado financie estas obras. El quinto desafío consiste en aumentar el gasto público social, especialmente en el Salvador, Guatemala, Honduras y Nicaragua, realizando un gran esfuerzo en todos los países por aumentar su eficacia. Lo anterior está vinculado con un 
sexto desafío que consiste en determinar la base institucional y los mecanismos requeridos para aumentar la eficacia del gasto público social. Ya existe una variedad de experiencias de desarrollo institucional en el istmo que pueden aprovecharse, aunque persiste la necesidad de evaluar de manera continua el impacto de los programas sociales que se están implementando y que se impulsarán en el futuro.

\section{b. Los desafíos tributarios}

Los cinco desafíos en el ámbito tributario incluyen, primero, aumentar la recaudación tributaria garantizando que aumente la carga tributaria. Esto implica, entre otras cosas, incluir a los sectores productivos más dinámicos dentro de la base tributaria. Segundo, debe fortalecerse la tributación directa, y particularmente el impuesto aplicado a la renta personal tomando en cuenta que su nivel es muy bajo cuando se compara con otros países. Ello requiere impulsar un debate acerca de la base del impuesto y su tratamiento (renta global, dual o cedular), su cobertura geográfica (mundial o territorial) y las tasas que se aplicarían (progresivas, una sola con un piso, o proporcionales), además del tratamiento de exenciones y exoneraciones.

Tercero, conviene mejorar la equidad de los sistemas tributarios asegurando que se perciban como justos y, en particular, que no faciliten la competencia desleal o la evasión. Enfrentar este desafío pasa por fortalecer la administración tributaria, cuya importancia debe destacarse fuertemente. Un cuarto desafío es mejorar las características técnicas de los impuestos para que no favorezcan unas actividades o sectores más que otros, que no promuevan la ineficiencia y que no aumenten el riesgo y la incertidumbre, desalentando la inversión. El quinto desafío consiste en lograr una convergencia y eventual armonización de los sistemas tributarios centroamericanos. La historia de amortización arancelaria junto con la voluntad de establecer una unión aduanera y de evitar una "guerra de incentivos" para atraer a la inversión extranjera son la base de este desafío.

\section{c) El desafío de implementar una estrategia de reformas}

El último gran desafío es de carácter político y es el prioritario. Consiste en implementar las reformas requeridas, especialmente las tributarias, para enfrentar los desafíos identificados previamente. La historia de la economía política de la tributación en América Central proporciona algunos elementos para la reflexión sobre este tema.

En primer lugar, plantea la conveniencia de concebir una estrategia de alianzas que puede ir más allá de los partidos y de los poderes legislativos - aunque sin quitarles su protagonismo, como base de la democracia - e incluir a otras organizaciones sociales y empresariales. La oposición fuerte y concentrada de las élites económicas a la tributación en el pasado, en particular, sugiere enfocarlas de manera selectiva, pudiéndose presumir menor oposición por parte de aquellas fracciones que simultáneamente están más distanciadas de la matriz agraria tradicional y que menos exigen exenciones y exoneraciones para crecer y competir. 
En segundo término, el contenido de las reformas podría generar menor oposición en la medida que favoreciera la equidad horizontal, trasmitiendo así un sentido de justicia y de ser contrarias a la competencia desleal. Integrar las reformas tributarias con otras reformas simultáneas - o previas - del gasto público para mejorar su transparencia y orientación, y con vistas a favorecer tanto la equidad como el crecimiento y la competitividad, también puede favorecer a la moral tributaria y crear la base para un contrato social más amplio sobre el contenido de la política fiscal.

En tercer lugar, el contexto internacional y la posición de actores externos pueden favorecer las reformas, aunque sin que ello sea necesariamente decisivo. Avanzar en este sentido puede significar obtener el apoyo de los organismos multilaterales de financiamiento y de la comunidad internacional; transmitirle un mensaje de reglas claras a empresas extranjeras que generalmente se ven más presionadas a cumplir con sus obligaciones tributarias que sus contrapartes nacionales (aún cuando acuden a técnicas más sofisticadas de evasión, como los precios de transferencia); ajustarse a normas internacionales como la obligación de la Organización Mundial de Comercio (OMC) de no exonerar del impuesto sobre la renta a los exportadores; y tomar en cuenta los dictámenes de las calificadoras de riesgo, que crecientemente identifican a una política fiscal sólida no sólo como condición para mantener la estabilidad macroeconómica sino también como requisito para asegurar la gobernabilidad.

Por último, la probabilidad de éxito de una estrategia de reforma será mayor si se impulsa como parte de una creciente conciencia nacional sobre la trascendencia de las finanzas públicas para el desarrollo de los países del istmo. Ello debería incluir una cada vez mayor moral tributaria y una creciente honestidad y eficacia en el uso de los recursos públicos, así como la sanción social de aquellos que buscan exenciones y exoneraciones a costa del bienestar nacional.

\section{Comentario de Néstor Avendaño a "La Política Fiscal en la encrucijada. El caso de América Central"}

Un estudio muy honesto y valiente. Así califico a "La Política Fiscal en la encrucijada - El caso de América Central”. En términos generales, se comprueba que la lógica económica de los Estados centroamericanos relega el gasto social por las crecientes y pesadas cargas del pago de las deudas internas y externas públicas, con la salvedad que Honduras y Nicaragua han recibido dos alivios de la deuda externa, la Iniciativa para Países Pobres Muy Endeudados y la Iniciativa de Reducción de la Deuda Multilateral. Aunque se reconocen los incrementos del gasto de educación y de atención primaria en salud, especialmente la materno-infantil, en todos los países de la subregión. 
Secuestionalatransparenciadelgastopúblico,principalmenteenlosámbitosdescentralizados. Sin embargo, pasan desapercibidas la calidad de la cooperación internacional y la calidad del gasto público para reducir la pobreza humana, así como la capacidad gubernamental para la ejecución de los proyectos de inversión pública, insuficiente en el caso de Nicaragua, cuando se fijan las metas decrecientes del déficit fiscal en los programas económicos que se establecen con el Fondo Monetario Internacional.

En nombre de los pobres y, de acuerdo con la jerga internacional, de los llamados pobres extremos - miserables, dirían Miguel de Cervantes y Víctor Hugo, con la riqueza del lenguaje - se han favorecido consultores y asesores nacionales y extranjeros, que desde escritorios gubernamentales elaboran estrategias de reducción de la pobreza y son remunerados con el nuevo endeudamiento externo. Por otro lado, se han desviado recursos del alivio de la deuda externa pública hacia el pago de la deuda interna pública en vez de ser asignados exclusivamente, tal como lo mandó el Grupo de los 7, a proyectos de educación, salud y saneamiento ambiental, y en forma adicional al gasto social que ya se registraba en el presupuesto antes de dicho alivio. Todo esto con el aval del Fondo Monetario Internacional, el gran coordinador que tuvo la misión de fortalecer el vínculo entre la reducción de la deuda externa y la reducción de la pobreza en ese extraño Club de 41 Países Pobres Muy Endeudados del Mundo.

Por el abuso que se ha cometido del nombre de los pobres, se han emprendido, por ejemplo en Nicaragua, procesos de "sinceramiento" del gasto público. Así le llaman en el argot multilateral y gubernamental, que no es nada más que clasificar correctamente los gastos en concepto de una planilla paralela financiada con recursos externos, trasladándola del gasto de capital al gasto de consumo del presupuesto nacional. Veo, con preocupación, que la cooperación internacional financia el consumo público en vez de asignarse preferentemente a la inversión pública. En particular, las donaciones de divisas líquidas destinadas al presupuesto con el fin de reducir la pobreza, son entregadas sin un destino específico que acerque a Nicaragua a las Metas del Milenio en 2015 y en las cuales observo más condicionalidades por un dólar comparadas a las de un dólar de libre disponibilidad prestado por organismos multilaterales.

Nuestros sistemas tributarios deberían llamarse sistemas de exenciones y exoneraciones tributarias. Dada la aún profunda regresividad de nuestros sistemas tributarios, el 70\% de los ingresos tributarios recaudados por los gobiernos centrales de los seis países del istmo centroamericano en 2006 corresponde a impuestos indirectos, las empresas denominadas como "grandes contribuyentes" deberían ser rebautizadas como "grandes retenedores" de impuestos indirectos. Pero los autores reconocen que la carga tributaria, o sea la proporción del Producto Interno Bruto del que se apodera el gobierno en concepto de impuestos, aumentó en todos los países del istmo no sólo por mayores tasas de crecimiento económico sino también por reformas tributarias parciales que han ampliado la base de contribuyentes y han mejorado la administración tributaria. Sin embargo, no es una simple coincidencia que los países más pobres, Nicaragua y Honduras, tengan las mayores cargas tributarias de $17 \%$ y $18 \%$ respectivamente en 2006. 
Los autores de esta obra son muy honestos al afirmar que "la oposición fuerte y concentrada de ciertas élites económicas no permitió que se desarrollara un sistema fiscal más completo y adecuado. En épocas más recientes algunas de estas élites han invertido en sectores privilegiados que no pagan impuestos y, por consiguiente, se oponen a que se fortalezca la tributación, a pesar de que estos sectores son los más dinámicos de la economía”. Además señalan que "persiste, en los partidos políticos, una dependencia financiera de los sectores económicos poderosos; y persiste una debilidad técnica en materia fiscal, especialmente tributaria”. Aquí terminan las citas. Los autores de esta obra honran la ética profesional.

Y este exuberante privilegio fiscal para una minoría, que se traduce en un atribulado gasto tributario de la mayoría, sustenta el segundo desafío tributario que exponen los autores en su obra: el fortalecimiento de la tributación directa. La responsabilidad social del empresario surge con el apropiado pago de sus impuestos directos, pero también debería buscarse una efectiva equidad en el pago de este impuesto, debería buscarse la equidad vertical en materia tributaria: el que tiene más, paga más, y el que tiene menos, paga menos. En mi opinión, deberían establecerse tasas impositivas diferenciadas para las microempresas, pequeñas empresas, medianas empresas y grandes empresas.

El primer desafío tributario que señalan los autores es aumentar la recaudación. ¿Con cuál objetivo? ¿Sólo para financiar el pago de la gran deuda social que tenemos con la población pobre de nuestros países centroamericanos? Además de proveer más recursos al Estado para iniciar una política de redistribución del ingreso, bastante incipiente unas veces e ignorada otras veces, las reformas tributarias que busquen una mayor recaudación de impuestos, por ejemplo a través de la eliminación de exoneraciones y exenciones excepto aquéllas que ordenan las Cartas Magnas de nuestros países, en mi entender deben basarse en genuinas estrategias de crecimiento y desarrollo económicos de nuestras naciones, que gocen de una efectiva participación de los gremios de productores y del respaldo de las fuerzas políticas del país. Estas estrategias no las hace el mercado y tengamos siempre presente que el presupuesto nacional es un instrumento en manos del Estado para dirigir ese proceso de desarrollo.

Comparto plenamente las premisas de los autores que completan los cinco desafíos tributarios y que apuntan a la reducción de la competencia desleal y de la evasión y la elusión tributarias con los fines de mejorar la equidad y financiar el gasto social; y al fortalecimiento de las instituciones recaudadoras de impuestos con el fin de disminuir los costos de la recaudación - ¿Cuánto cuesta al Estado recaudar, por ejemplo, 1 quetzal, 1 lempira, 1 colón, 1 córdoba o 1 dólar en concepto de impuestos? -; y la armonización de los sistemas tributarios centroamericanos, no sólo con el afán de impedir la "guerra de incentivos" para atraer la inversión extranjera y avanzar en la unión aduanera, sino también en el logro de la homogeneidad de la política macroeconómica de los países del istmo. Sin embargo, agrego para la consideración de los autores y de las autoridades públicas, dos premisas más: que se eliminen totalmente todos los beneficios tributarios que descansan en exoneraciones y exenciones de impuestos indirectos y se analice la posibilidad de establecer beneficios tributarios temporales en el marco de la imposición directa - por ejemplo, la exoneración temporal del pago del impuesto sobre la renta de las empresas siempre y cuando el empresario presente un plan de inversión para expandir la infraestructura productiva y, 
así, facilite la aceleración de la tasa de crecimiento económico en cada uno de nuestros países, con la debida supervisión estatal -; y que se dote, por decreto presidencial, de la mayor fuerza política a los entes recaudadores de impuestos con el fin de garantizar el apropiado pago de los impuestos.

Y en cuanto a los seis desafíos que enfrenta el gasto público, los autores enfatizan en una mayor transparencia y un mejor control en el uso de los recursos públicos mediante el fortalecimiento de las contralorías generales, pero he de agregar que no basta sólo la transparencia en la ejecución del presupuesto. También propongo a los autores y a las autoridades públicas que es necesaria la transparencia en la formulación del presupuesto con el propósito de evitar indebidas asignaciones de los recursos del Tesoro. Por ejemplo, en el caso de Nicaragua, los recursos provenientes de los alivios de la deuda externa en vez de haber sido destinados a los pobres fueron asignados al pago de onerosos e ilícitos endeudamientos internos. Así se impediría lo que he llamado la corrupción "lícita" en el sector público, una vez que la desviación ha sido aprobada por la Asamblea Nacional.

El llamado de los autores a formular y aprobar presupuestos multianuales requiere de una atención gubernamental apropiada, porque la efectividad no sólo de las políticas sociales sino también de la cooperación internacional destinada al presupuesto con el ánimo de reducir la pobreza humana, no es posible con presupuestos de corto plazo. La descentralización del gasto público se ha iniciado con las transferencias de ingresos tributarios de los gobiernos centrales hacia los gobiernos municipales, pero es urgente descentralizar tanto el poder político como los recursos humanos calificados que se concentran en los gobiernos centrales. También apoyo el mensaje de los autores de incrementar el gasto social y su eficacia, así como la inversión en infraestructura económica, y de fortalecer no sólo nuestras instituciones públicas sino también nuestro andamiaje legal para mejorar y maximizar los resultados de las experiencias administrativas apropiadas con la reducción de la pobreza y el incremento del bienestar social.

En conclusión, los autores nos muestran las necesidades de impulsar el crecimiento económico equitativo con la aplicación de reformas tributarias apropiadas, de una mayor transparencia en la formulación y la ejecución del presupuesto nacional, y de una mejora de la calidad de la cooperación internacional y de la eficacia del gasto público. En otras palabras, los autores nos muestran la necesidad de mejorar la gobernabilidad en cada uno de nuestros países centroamericanos.

Finalmente, felicito a cada uno de los miembros del equipo de investigación de esta obra, al Consejo Asesor del Instituto Centroamericano de Estudios Fiscales (ICEFI), a la Junta Directiva de Guatemala, a los miembros de los equipos de producción y difusión de la obra, a la Supervisora General Sra. Iliana Peña, Directora de Administración y Finanzas del ICEFI y en especial al Coordinador General, Sr. Juan Alberto Fuentes Knight, Director Ejecutivo del ICEFI. 


\section{Nicaragua: Historia de inmigrantes}

Autor: Eddy Kühl Aráuz

\section{Comentario de José Luis Rocha}

"Nicaragua: Historia de inmigrantes", el último libro del prolífico empresario e historiador Eddy Kühl, es uno de los textos de historia que más aporta a la comprensión de Nicaragua. Con meticulosidad de orfebre, Kühl recoge y engarza información sobre los flujos humanos que han hecho de este país lo que es, mostrando su influjo en los grandes acontecimientos históricos y señalando su impacto en el desarrollo económico, los tejidos sociales y las instituciones culturales nicaragüenses. Quizás sin proponerse una polémica -al menos no de manera explícita-, este libro camina a contrapelo de una compleja urdimbre de supuestos, mitos y lugares comunes que fundan una falsa imagen de esa nación que es Nicaragua. En primer lugar, se contrapone a la idea de una nación compuesta por un grupo homogéneo de pobladores, promedio o tensión de dos grandes grupos humanos. Se desvanece, por tanto, el hombre dual de Pablo Antonio

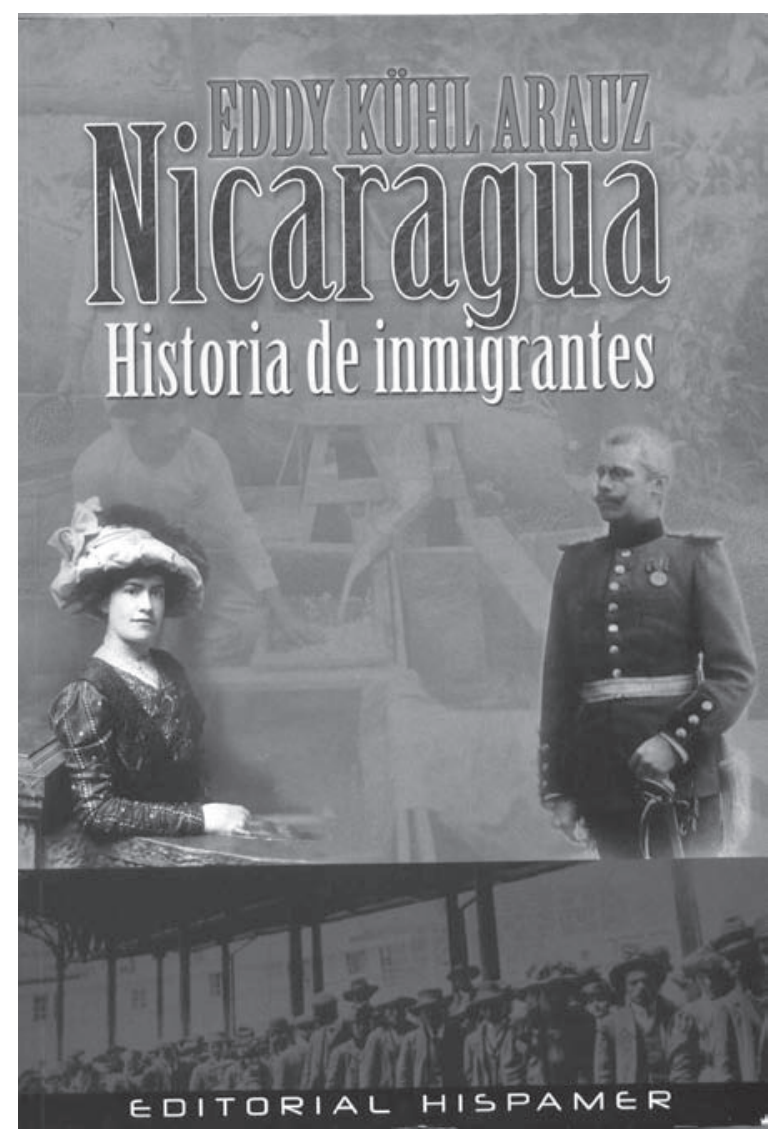
Cuadra -medio indio y medio español - para abrir paso a muchos grupos de hombres pluriculturales y variopintos: chorotegas, alemanes, españoles, franceses, suecos, africanos, japoneses, chinos, garífonas, británicos, italianos, belgas, irlandeses, árabes, judíos, etc. La nicaraguanidad no puede ser reducida a un grupo, o a un par de grupos. Tampoco puede ser promediada. Nicaragua, como todos los países, es una historia de inmigrantes. Por eso resulta significativo que el libro se titule "Nicaragua: Historia de inmigrantes", y no "Historia de las migraciones hacia Nicaragua".

En segundo lugar, el alucinante recorrido que realiza Eddy Kühl nos recuerda que hemos llegado a este punto de nuestra historia tras una larga serie de olas migratorias. No estamos en el mismo punto de partida. ¿Será este un punto de llegada? Los incesantes movimientos poblacionales parecen dar un rotundo "no" como respuesta. La nicaraguanidad no es estática, 
como a muchos gusta creer. Experimenta graduales o incluso violentas transformaciones. El libro de Kühl permite un seguimiento de los migrantes de acuerdo a su origen, pero también facilita información para un seguimiento cronológico, de modo que el lector pueda captar el impacto de los distintos grupos y personajes en el tiempo.

En tercer lugar, los relatos que componen este gran relato de los inmigrantes revelan constantemente que los países se enriquecen con los migrantes. Los migrantes son ganancia para los países de acogida. Kühl proporciona un erudito recuento de sus contribuciones a los distintos sectores productivos -incluso con ejemplos que parecen provenir del realismo mágico, como es el caso del terrocarril, un ferrocarril sin rieles, que inventaron los alemanes en Matagalpa-, a los antiguos vínculos con el comercio internacional gourmet -como es el caso de la Casa Meniere-, a la historiografía, a la medicina, a la arquitectura y a la literatura, entre otros de los muchos campos en los cuales la contribución de los inmigrantes ha sido determinante, plausible y memorable.

Todo el libro está salpicado de curiosas e incluso hilarantes historias -algunas sobre visitantes ilustres, como Mark Twain y Howard Hughes- para que su lectura resulte más amena y atractiva, además de contundentemente instructiva. Por eso agradecemos a Eddy Kühl que su aporte a la historia nos rescate de esa ignorancia que se ignora a sí misma tan aborrecida por José Coronel Urtecho. 